\title{
Nucleosynthesis and mixing in rotating AGB stars at low metallicity
}

\section{T. Decressin ${ }^{\star a}$, C. Charbonnel ${ }^{a b}$}

${ }^{a}$ Geneva Observatory, 51, chemin des Maillettes, 1290 Sauverny, Switzerland

${ }^{b}$ LA2T, OMP, CNRS UMR 5572, 14, Av. E. Belin, 31400 Toulouse, France

E-mail: Thibaut.Decressin@obs.unige.ch,

Corinne. Charbonneldobs.unige.ch

\begin{abstract}
Models of rotating stars at $Z=5 \times 10^{-4}$ and $10^{-5}$ with masses between 2.5 and $7 \mathrm{M}_{\odot}$ are computed from the pre-main sequence up to the end of the asymptotic giant branch phase (AGB). We discuss the impact of rotation on nucleosynthesis. We show that it plays a major role during the phase of central He-burning. Rotational-induced mixing transports indeed freshly produced carbon and oxygen into the H-burning shell where some new primary elements are synthesized. In the most massive early-AGB stars, those elements are revealed at the surface during the second dredge-up event. We also follow the changes in surface and wind composition due to the interplay between third dredge-up and hot bottom burning during the TP-AGB phase. We present the final stellar yields and discuss our predictions in the context of the chemical anomalies seen in galactic globular clusters.
\end{abstract}

International Symposium on Nuclear Astrophysics - Nuclei in the Cosmos - IX

June 25-30 2006

CERN, Geneva, Switzerland

${ }^{*}$ Speaker. 


\section{Physical inputs of the stellar models}

Both rotating and standard models for stars with initial masses between 2.5 to $7 \mathrm{M}_{\odot}$ are computed at $Z=5 \times 10^{-4}([\mathrm{Fe} / \mathrm{H}]=-1.6)$. The metallicity dependence of our theoretical predictions is investigated for $7 \mathrm{M}_{\odot}$ for which we compute a model at $Z=10^{-5}$. The initial rotation velocity of our models is taken equal to $300 \mathrm{~km} \cdot \mathrm{s}^{-1}$.

During main sequence, we follow in a self-consistent way the transport of angular momentum and of chemicals through meridional circulation and turbulence using the prescriptions by [1] and [2] (see also [3]). During He-burning, only local angular momentum conservation is used as structural changes now dominate the momentum evolution. Rotational mixing is stopped during AGB phase.

During the AGB phase mass loss is switched from Reimers prescription [4] to Blöcker one [5] (with $\eta=0.05)$ with an explicit $\sqrt{Z}$ dependence. Diffusive overshooting [6] $\left(f_{\text {over }}=0.016\right)$ is added at the bottom of the convective envelope during the TP-AGB phase.

\section{Pre-AGB phases}
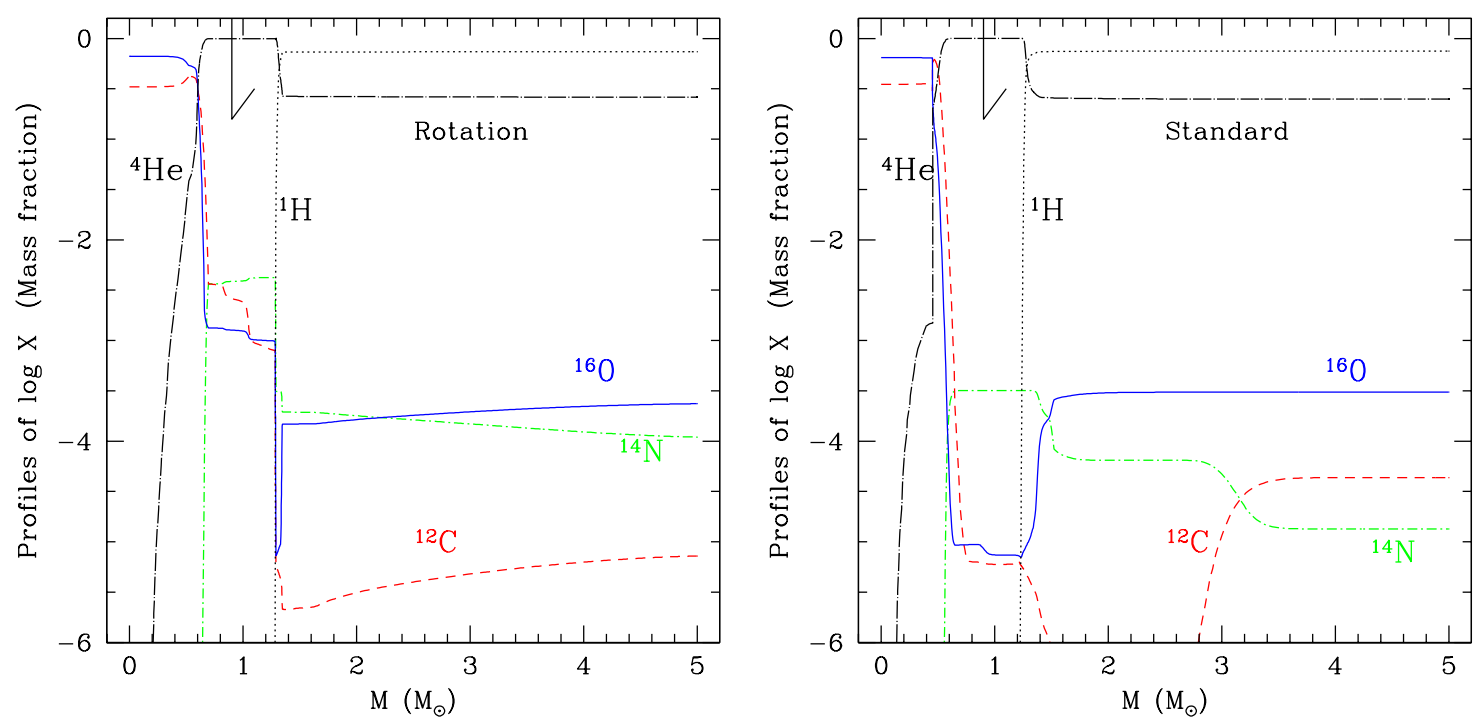

Figure 1: Chemical profiles of selected nuclei for rotating (left) and standard (right) $5 \mathrm{M}_{\odot}$ stars at the end of central He-burning phase. The arrows indicate the deepest penetration of the convective envelope during the second dredge-up phase.

During the pre-AGB phases, rotation-induced mixing modifies the chemical structure of the star with respect to the standard predictions. One striking effect can be seen in Fig. 1 where the chemicals profiles of some key elements at the end of the central He-burning phase are displayed for the $5 \mathrm{M}_{\odot}$ for rotating and standard cases. The non rotating models present a sharp CO core surrounded by a He-rich layer where the signatures of the $\mathrm{CNO}$ cycle are well visible. On the other hand, the rotating models show the signatures of an important diffusion of $\mathrm{C}$ and $\mathrm{O}$ from the core 

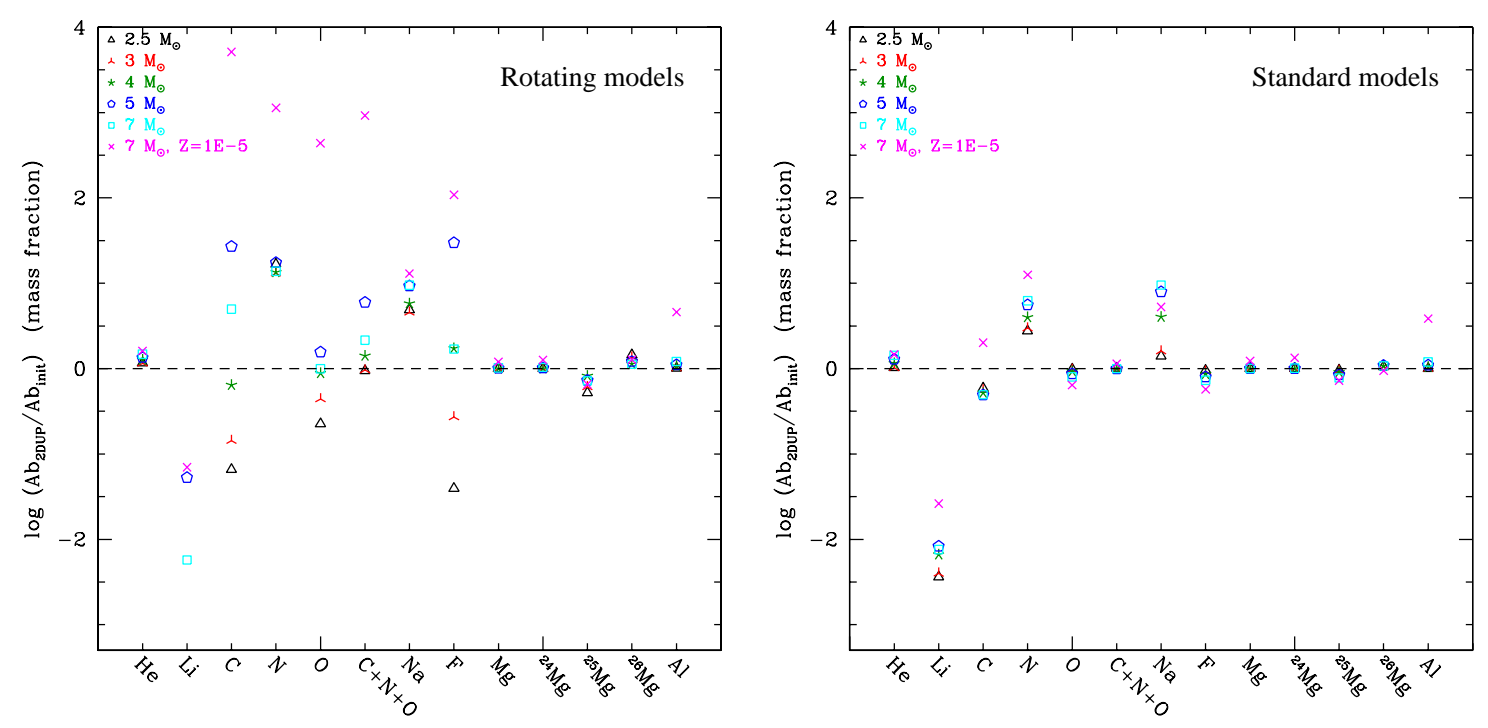

Figure 2: Ratio of surface abundances after 2DUP completion to initial ones for the rotating (left) and standard model (right).

to the more external layers. Part of this $\mathrm{C}$ and $\mathrm{N}$ is converted into $\mathrm{N}$ in the He-rich layers where some protons are also mixed.

For these patterns to be revealed at the surface requires the occurrence of the second dredge-up (2DUP) event. This happens only in the higher mass stars $\left(M \geq 4 \mathrm{M}_{\odot}\right.$; in less massive stars the convective envelope sinks only into the $\mathrm{H} / \mathrm{He}$ discontinuity during the 2DUP). In these objects the convective envelope then sinks into the He-rich layers as shown in Fig. 1 where the arrows indicate the deepest penetration of the convective envelope during the 2DUP phase.

Fig. 2 illustrates the surface abundance variations after the 2DUP for all the stellar masses considered, in both the standard and rotating cases. Only the elements lighter than $\mathrm{Mg}$ are affected. For the low mass stars, only the layers where the CNO cycle operates outside of the He-rich layers are reached by the deepening convective envelope at that phase. More massive rotating stars show a strong increase of primary $\mathrm{C}, \mathrm{O}, \mathrm{N}$ and $\mathrm{F}$ abundance at the surface after the 2DUP as those elements are synthesized in the He-rich layers before being dredged by the convective envelope. The $7 \mathrm{M}_{\odot}$ rotating model at $Z=10^{-5}$ shows stronger abundance variations.

\section{Winds on the TPAGB phase and comparison with globular cluster stars}

Table 1 presents some structural parameters of AGB models. Core is more massive in rotating models at the beginning of the AGB phase as some mixing have extended this core during central He-burning phase. Recurring third dredge-up (3DUP) prevent strong increase during TPAGB as the final mass of the remnant is only a few $0.1 \mathrm{M}_{\odot}$ heavier.

Now we turn our attention to the chemical changes for low mass stars $\left(M \leq 3 \mathrm{M}_{\odot}\right)$. The surface abundance patterns obtained during the 2DUP is only modified by recurring 3DUP events (see Fig. 3). It mainly increases the carbon abundance. Fluorine abundance raises after each 3DUP and a positive production if found in the mean composition of winds. 


\begin{tabular}{c|cccc|cccc}
\hline & \multicolumn{5}{|c|}{ Rotating models } & \multicolumn{4}{c}{ Standard models } \\
$\mathrm{M}\left(\mathrm{M}_{\odot}\right)$ & $\mathrm{M}_{2 \text { DUP }}\left(\mathrm{M}_{\odot}\right)$ & $\mathrm{N}_{\mathrm{TP}}$ & $\lambda_{\max }$ & $\mathrm{M}_{\mathrm{f}}\left(\mathrm{M}_{\odot}\right)$ & $\mathrm{M}_{2 \text { DUP }}\left(\mathrm{M}_{\odot}\right)$ & $\mathrm{N}_{\mathrm{TP}}$ & $\lambda_{\max }$ & $\mathrm{M}_{\mathrm{f}}$ \\
\hline \hline 2.5 & 0.72 & 24 & 0.73 & 0.78 & 0.67 & 30 & 0.68 & 0.74 \\
3 & 0.84 & 24 & 0.82 & 0.86 & 0.77 & 30 & 0.77 & 0.83 \\
4 & 0.86 & 33 & 0.80 & 0.89 & 0.86 & 27 & 0.85 & 0.88 \\
5 & 0.89 & 49 & 0.79 & 0.91 & 0.90 & 30 & 0.88 & 0.92 \\
7 & 1.07 & $>28$ & 0.86 & - & 1.01 & $>15$ & 1.00 & - \\
\hline
\end{tabular}

Table 1: Structural parameter of TPAGB phase for rotating and standard models at $Z=5 \times 10^{-4}$ : core mass after 2DUP completion, number of thermal pulses, maximum dredge-up efficiency and final core mass
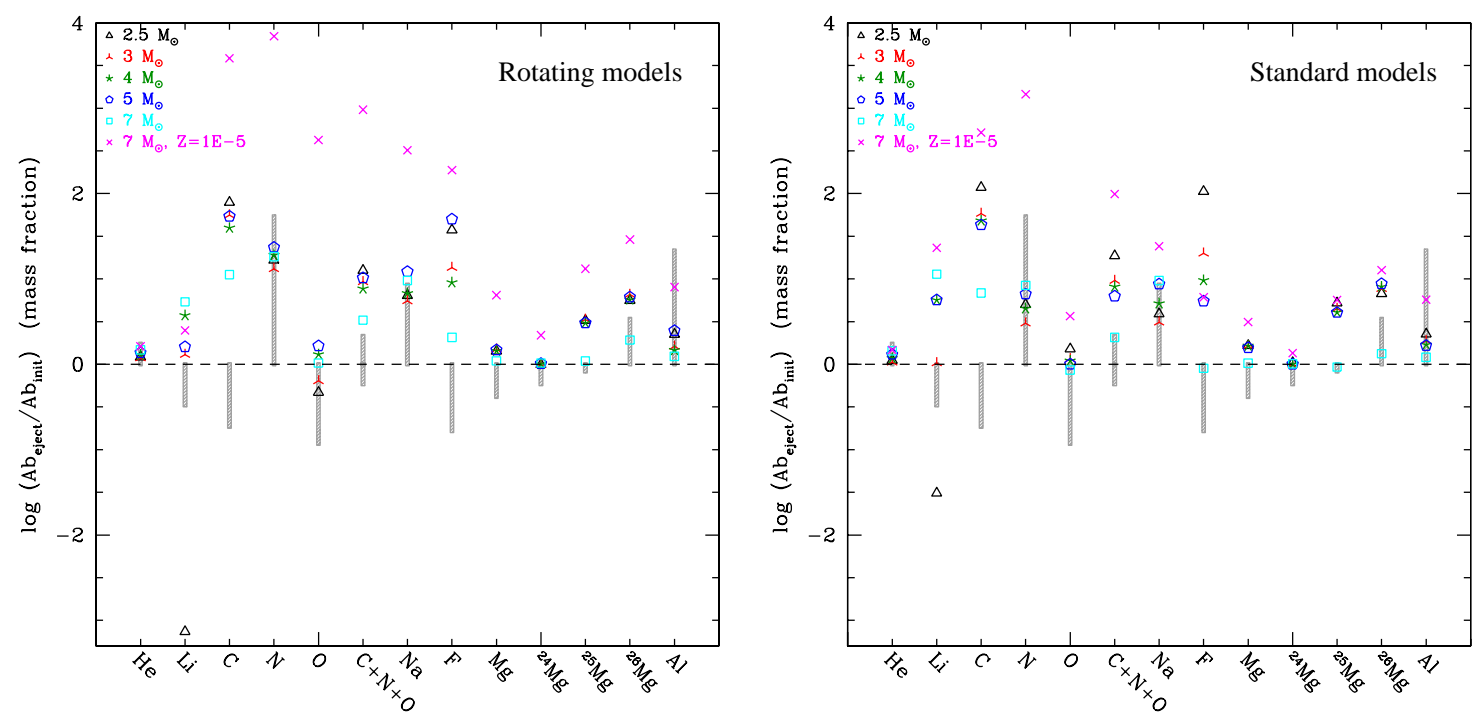

Figure 3: Ratio of mean abundances in stellar winds to initial composition for rotating (left) and standard (right) models. The typical abundance variations seen in globular cluster stars are indicated by grey bars.

In massive TP-AGB stars $\left(M \geq 4 \mathrm{M}_{\odot}\right)$ the bottom of the convective envelope reaches temperatures between 50 and $70 \times 10^{6} \mathrm{~K}$, and hot bottom burning (HBB) occurs. It converts part of the fresh primary carbon and neon produced within the convective thermal pulses into nitrogen and sodium respectively. However the $\mathrm{Na}$ abundance stays close to the level obtained after 2DUP. $\mathrm{Mg}$ and $\mathrm{Al}$ surface abundance have a similar evolution in the rotating and standard cases because they were not modified in the pre TP-AGB phases. ${ }^{25} \mathrm{Mg}$ and ${ }^{26} \mathrm{Mg}$ increase due to their synthesis within the thermal pulses followed by 3DUP. As ${ }^{24} \mathrm{Mg}$ is not consumed the total $\mathrm{Mg}$ abundance increases.

AGB are often thought to be responsible of abundance anomalies seen exhibited by globular cluster (GC) stars (see [7] in these proceedings). In Fig. 3 we thus compare our predictions for the wind composition with the GC data. The C-N anticorrelation [8] is obtained in none of our models as AGB winds display strong carbon and nitrogen enrichment due respectively to the 3DUP and HBB. Low-mass rotating AGBs slightly deplete $\mathrm{O}$ as the results of rotational mixing during the pre-AGB phase. However this $\mathrm{O}$ depletion is much too small to explain the oxygen data shown in GCs. In all the models the raise in the $\mathrm{Na}$ abundance well matches the observational trends. Last 
but not least the theoretical wind composition is at odd with the Mg-Al anticorrelation seen in GCs as all our AGB stars produce magnesium together with little quantities of aluminum. In addition the theoretical magnesium isotopic composition that does not recover the one found by [10] $\left({ }^{24} \mathrm{Mg}\right.$ appears to be depleted in observed GC stars).

\section{Conclusion}

Rotation has several effects on the chemical patterns of low- and intermediate-mass stars. During the central He-burning phase in particular it leads to the mixing of the He-burning products into the He-rich layers where some protons are also transported from the radiative envelope. There $\mathrm{N}$ is synthesized. This effect is dominant in high-mass AGB stars whose convective envelope penetrates into those deep layers during the 2DUP. Very low metallicity stars are very sensitive to this mechanism: due to their low initial abundance the dilution is stronger.

The 3DUP and HBB modify the patterns resulting from the 2DUP. In low mass AGBs the evolution of the surface abundances of nitrogen, oxygen is dominated by the 2DUP whereas their carbon abundance raises mainly thank to the 3DUP on the TP-AGB. The same is true for massive AGB except that nitrogen increases also thanks to HBB on the TP-AGB.

When compared to GC data one finds that only the Na increase can be reproduced by all the models; however only the low-mass rotating AGB stars slightly deplete O. None of the other observed patterns is reproduced by the AGB models. We must thus conclude that these stars are probably not responsible for the GC abundance anomalies.

\section{References}

[1] Zahn J.-P., 1992, $A \& A, 265,115$

[2] Maeder A., Zahn J.-P., 1998, A\&A, 334, 1000

[3] Palacios, A., Charbonnel, C., Talon, C., Siess, L., 2005, A\&A, 453, 261

[4] Reimers D., 1975, Mem. Soc. Roy. Sci. Liège, 6th Ser., 8, 369

[5] Blöcker T., 1995, A\&A, 297, 727

[6] Herwig F., 2000, $A \& A, 360,952$

[7] Charbonnel C., 2006, in proceedings of Nuclei in the Cosmos - IX

[8] Carretta E., Gratton R.G. Lucatello S. et al., 2005, A\&A, 433, 597

[9] Gratton R.G, Bonifacio P. \& Bragaglia A., 2001 A\&A, 369, 87

[10] Yong D., Lambert D. L., Ivans I. I., 2003, ApJ, 599, 1357

[11] Pasquini L., Bonifacio P., Molaro P. et al., 2005, A\&A, 441, 549

[12] Smith V.V., Cunha K., Ivans I.I. et al., 2005 ApJ, 633, 392 